\title{
The Finite Spectrum of Fourth-Order Boundary Value Problems with Transmission Conditions
}

\author{
Fang-zhen Bo ${ }^{1}$ and Ji-jun $\mathrm{Ao}^{2}$ \\ ${ }^{1}$ Normal College, Hohhot Vocational College, Hohhot 010051, China \\ ${ }^{2}$ College of Sciences, Inner Mongolia University of Technology, Hohhot 010051, China
}

Correspondence should be addressed to Ji-jun Ao; george_ao78@sohu.com

Received 15 February 2014; Accepted 4 June 2014; Published 24 June 2014

Academic Editor: Paul W. Eloe

Copyright (C) 2014 F.-z. Bo and J.-j. Ao. This is an open access article distributed under the Creative Commons Attribution License, which permits unrestricted use, distribution, and reproduction in any medium, provided the original work is properly cited.

A class of fourth-order boundary value problems with transmission conditions are investigated. By constructing we prove that these class of fourth order problems consist of finite number of eigenvalues. Further, we show that the number of eigenvalues depend on the order of the equation, partition of the domain interval, and the boundary conditions (including the transmission conditions) given.

\section{Introduction}

Boundary value problems with finite spectrum have been studied recently [1-10]. These problems come from Atkinson's statement in his book "Discrete and Continuous Boundary Problems" [1] in 1964. In 2001 Kong et al. [2] constructed, for each positive integer $m$, a class of Sturm-Liouville problems with separated and coupled boundary conditions whose spectrum consists of exactly $m$ eigenvalues. This is the first time that the finite spectrum results about the boundary value problems are proven. Then in 2009, Kong et al. [3] found the matrix representations of these kinds of S-L problems with finite spectrum. In 2011 and 2012, Ao et al. generalized the finite spectrum results and corresponding matrix representations to S-L problems with transmission conditions $[4,6]$. And soon the finite spectrum of fourth-order boundary value problems and the matrix representations are also given by Ao et al. $[8,9]$.

In recent years, boundary value problems with transmission conditions or eigenparameter dependent boundary conditions have been an important research topic for their applications in physics [11-14]. The asymptotic behaviors of eigenvalues and eigenfunctions of a class of fourth-order differential operators with transmission conditions have been studied in [13]. Following [13] and together with $[4,10]$, we will consider fourth-order boundary value problems with transmission conditions in this paper. We try to show that these problems also have finite spectrum though the analysis is more complicated than before. The results also show that the number of eigenvalues of these kinds of fourth-order boundary value problems with transmission conditions is dependent on the order of the equation, partition of the domain interval, and the boundary conditions (including the transmission conditions) given. The key to this analysis is still an iterative construction of the characteristic function.

\section{Notation and Preliminaries}

We consider the fourth-order boundary value problems (BVPs) consisting of the equation

$$
\begin{gathered}
\left(p y^{\prime \prime}\right)^{\prime \prime}+q y=\lambda w y, \quad \text { on } J=(a, c) \cup(c, b), \\
c \in(a, b), \quad \text { with }-\infty<a<b<+\infty
\end{gathered}
$$

together with boundary conditions

$$
\begin{gathered}
A Y(a)+B Y(b)=0, \\
Y=\left(\begin{array}{c}
y \\
y^{\prime} \\
p y^{\prime \prime} \\
\left(p y^{\prime \prime}\right)^{\prime}
\end{array}\right), \quad A, B \in M_{4}(\mathbb{C}),
\end{gathered}
$$


and transmission conditions

$$
C Y(c-)+D Y(c+)=0,
$$

where $c$ is an inner discontinuous point, $M_{4}(\mathbb{C})$ denotes the set of square matrices of order 4 over the complex numbers $\mathbb{C}$, and $C, D$ are real valued $4 \times 4$ matrices satisfying $\operatorname{det}(C)=$ $\rho>0, \operatorname{det}(D)=\theta>0$. Here $\lambda$ is the spectral parameter, and the coefficients satisfy the minimal condition

$$
r=\frac{1}{p}, q, w \in L(J, \mathbb{C}),
$$

where $L(J, \mathbb{C})$ denotes the complex valued functions which are Lebesgue integrable on $J$. Condition (4) is minimal in the sense that it is necessary and sufficient for all initial value problems of (1) to have unique solutions on $J$; see $[15,16]$.

By introducing the quasiderivatives $u_{1}=y, u_{2}=y^{\prime}, u_{3}=$ $p y^{\prime \prime}, u_{4}=\left(p y^{\prime \prime}\right)^{\prime}$. Then (1) can be written as the following system representation $[17,18]$ :

$$
\begin{gathered}
u_{1}^{\prime}=u_{2}, \quad u_{2}^{\prime}=r u_{3}, \quad u_{3}^{\prime}=u_{4}, \\
u_{4}^{\prime}=(\lambda w-q) u_{1}, \quad \text { on } J .
\end{gathered}
$$

Remark 1. Note that condition (4) does not restrict the sign of any of the coefficients $r, q, w$. Also, each of $r, q, w$ is allowed to be identically zero on subintervals of $J$. If $r$ is identically zero on a subinterval $I$, then there exists a solution $y$ which is identically zero on $I$, but one of its quasiderivatives $u_{2}=y^{\prime}$, $u_{3}=p y^{\prime \prime}, u_{4}=\left(p y^{\prime \prime}\right)^{\prime}$ may be a nonzero constant function on $I$.

Definition 2. By a trivial solution of (1) on a subinterval $I \subset J$ we mean a solution $y$ which is identically zero on $I$ and whose quasiderivatives $u_{2}=y^{\prime}, u_{3}=p y^{\prime \prime}, u_{4}=\left(p y^{\prime \prime}\right)^{\prime}$ are also identically zero on $I$.

We comment on the self-adjoint expressions of the fourth-order boundary value problems (1)-(3) briefly. Without the transmission condition (3), it is well known $[19,20]$ that the self-adjoint $\mathrm{BCs}$ of fourth-order problem are the conditions (2) where the matrices $A, B$ satisfy

$$
\operatorname{rank}(A: B)=4, \quad A E_{4} A^{*}=B E_{4} B^{*}
$$

with $E_{4}$ denoting the symplectic matrix

$$
E_{4}=\left(\begin{array}{cccc}
0 & 0 & 0 & -1 \\
0 & 0 & 1 & 0 \\
0 & -1 & 0 & 0 \\
1 & 0 & 0 & 0
\end{array}\right)
$$

However, while the transmission condition (3) occurs in the problem, the self-adjoint conditions are alternatively given by

$$
\begin{gathered}
\operatorname{rank}(A: B)=4, \quad A E_{4} A^{*}=B E_{4} B^{*}, \\
\frac{1}{\rho} C E_{4} C^{*}=\frac{1}{\theta} D E_{4} D^{*}
\end{gathered}
$$

with $E_{4}$ given as in (7).
An important class of self-adjoint boundary conditions is the separated condition. It has the following canonical representation $[19,20]$ :

$$
\begin{aligned}
& A_{1} u+A_{2} v=0 \quad \text { at } x=a \\
& B_{1} u+B_{2} v=0 \quad \text { at } x=b,
\end{aligned}
$$

where $u=\left(u_{1}, u_{2}\right)^{T}, v=\left(u_{3}, u_{4}\right)^{T}$, and $A_{1}, A_{2}, B_{1}, B_{2}$ are $2 \times 2$ matrices such that $A_{1} E_{2} A_{2}^{*}-A_{2} E_{2} A_{1}^{*}=0, B_{1} E_{2} B_{2}^{*}-$ $B_{2} E_{2} B_{1}^{*}=0$, with $E_{2}=\left(\begin{array}{cc}0 & -1 \\ 1 & 0\end{array}\right)$, and the $2 \times 4$ matrices $\bar{A}=$ $\left(A_{1}: A_{2}\right), \bar{B}=\left(B_{1}: B_{2}\right)$ have rank 2 .

Lemma 3. Let (4) hold and let $\Phi(x, \lambda)=\left[\phi_{i j}(x, \lambda)\right]$ denote the fundamental matrix of the system (5) determined by the initial condition $\Phi(a, \lambda)=I$. Then a complex number $\lambda$ is an eigenvalue of the fourth-order problem (1)-(3) if and only if

$$
\Delta(\lambda)=\operatorname{det}[A+B \Phi(b, \lambda)]=0 .
$$

Proof. Suppose $\Delta(\lambda)=0$. Then $[A+B \Phi(b, \lambda)] \widetilde{C}=\mathbf{0}$ has a nontrivial vector solution. Solve the initial value problem

$$
\begin{aligned}
& Y^{\prime}=\left(\begin{array}{cccc}
0 & 1 & 0 & 0 \\
0 & 0 & \frac{1}{p} & 0 \\
0 & 0 & 0 & 1 \\
\lambda w-q & 0 & 0 & 0
\end{array}\right) Y, \\
& Y=\left(\begin{array}{c}
y \\
y^{\prime} \\
p y^{\prime \prime} \\
\left(p y^{\prime \prime}\right)^{\prime}
\end{array}\right) \text { on } J, \quad Y(a)=\widetilde{C} .
\end{aligned}
$$

Then $Y(b)=\Phi(b, \lambda) Y(a)$ and $[A+B \Phi(b, \lambda)] Y(a)=\mathbf{0}$.

From this, it follows that the top component of $Y$, say, $y$, is an eigenfunction of the fourth-order problem (1)-(3); this means that $\lambda$ is an eigenvalue of this problem. Conversely, if $\lambda$ is an eigenvalue and $y$ is an eigenfunction of $\lambda$, then

$$
Y=\left(\begin{array}{c}
y \\
y^{\prime} \\
p y^{\prime \prime} \\
\left(p y^{\prime \prime}\right)^{\prime}
\end{array}\right)
$$

satisfies $Y(b)=\Phi(b, \lambda) Y(a)$ and consequently $[A+$ $B \Phi(b, \lambda)] Y(a)=\mathbf{0}$. Since $Y(a)=\mathbf{0}$ would imply that $y$ is the trivial solution in contradiction to it being an eigenfunction, we have that $\operatorname{det}[A+B \Phi(b, \lambda)]=0$.

Remark 4. In the proof of Lemma 3, the transmission condition (3) does not affect the proof and only makes the quasiderivatives as piecewise functions. This will be given later (see Remark 10).

Next we find a formula for $\Delta(\lambda)$ which highlights its dependence on $\lambda$ and on the matrices $A, B$. 
Lemma 5. Let (2) hold and let $\Phi(x, \lambda)=\left[\phi_{i j}(x, \lambda)\right]$ denote the fundamental matrix of the system (5) determined by the initial condition $\Phi(a, \lambda)=I$. Then the characteristic function $\Delta(\lambda)=\operatorname{det}[A+B \Phi(b, \lambda)]$ can be written as

$$
\begin{aligned}
\Delta(\lambda)= & \operatorname{det}(A)+\operatorname{det}(B)+\sum_{i=1}^{4} \sum_{j=1}^{4} c_{i j} \phi_{i j} \\
& +\sum_{1 \leq i, j, k, l \leq 4, j \neq l} d_{i j k l} \phi_{i j} \phi_{k l} \\
& +\sum_{1 \leq i, j, k, l, m, n \leq 4, j \neq l \neq n} e_{i j k l m n} \phi_{i j} \phi_{k l} \phi_{m n},
\end{aligned}
$$

where $c_{i j}, 1 \leq i, j \leq 4, d_{i j k l}, 1 \leq i, j, k, l \leq 4, j \neq l, e_{i j k l m n}$, $1 \leq i, j, k, l, m, n \leq 4, j \neq l \neq n$ are constants which depend only on the matrices $A$ and $B$.

Proof. Firstly, for any $A=\left(a_{i j}\right), B=\left(b_{i j}\right) \in M_{4}(\mathbb{C})$, it is obvious that

$$
\operatorname{det}(A+B)=\operatorname{det}(A)+\operatorname{det}(B)+P(A, B),
$$

where $P(A, B)$ denotes the sum of the possible products of the elements belonging to different rows and different columns in matrices $A$ and $B$.

Hence we have

$$
\begin{aligned}
\Delta(\lambda) & =\operatorname{det}[A+B \Phi(b, \lambda)] \\
& =\operatorname{det}(A)+\operatorname{det}(B \Phi(b, \lambda))+P(A, B \Phi(b, \lambda))
\end{aligned}
$$

Since $\Phi(a, \lambda)=I$, then $\operatorname{det}(\Phi(b, \lambda))=1$, and $P(A, B \Phi(b, \lambda))$ can be written in the form

$$
\begin{aligned}
P(A, B \Phi(b, \lambda))= & \sum_{i=1}^{4} \sum_{j=1}^{4} c_{i j} \phi_{i j}+\sum_{1 \leq i, j, k, l \leq 4, j \neq l} d_{i j k l} \phi_{i j} \phi_{k l} \\
& +\sum_{1 \leq i, j, k, l, m, n \leq 4, j \neq l \neq n} e_{i j k l m n} \phi_{i j} \phi_{k l} \phi_{m n},
\end{aligned}
$$

where $c_{i j}, 1 \leq i, j \leq 4, d_{i j k l}, 1 \leq i, j, k, l \leq 4, j \neq l, e_{i j k l m n}$, $1 \leq i, j, k, l, m, n \leq 4, j \neq l \neq n$ are constants which depend only on the matrices $A$ and $B$.

Then we can conclude that (13) is followed.

Corollary 6. Consider the problem (1) with separated selfadjoint BCs (9). Then the characteristic function $\Delta(\lambda)$ of the problem (1), (9) has the form

$$
\Delta(\lambda)=\sum_{1 \leq i, j, k, l \leq 4, j \neq l} d_{i j k l} \phi_{i j} \phi_{k l} .
$$

Proof. Note that the third and fourth row of $A$ and the first and second row of $B$ in BCs (2) are zero, and hence we have that $\operatorname{det}(A)=0, \operatorname{det}(B)=0$, and the third part and the fifth part in (13) vanish; then the result follows.

The fourth-order problem (1)-(3), or equivalently (5), (2), and (3), is said to be degenerate if in (13) either $\Delta(\lambda) \equiv 0$ for all $\lambda \in \mathbb{C}$ or $\Delta(\lambda) \neq 0$ for every $\lambda \in \mathbb{C}$.

\section{Fourth-Order Problems with Finite Spectrum}

In this section we assume that (2) holds and there exists a partition of the interval $J$

$$
\begin{gathered}
a=a_{0}<a_{1}<a_{2}<\cdots<a_{2 m}<a_{2 m+1}=c, \\
c=b_{0}<b_{1}<b_{2}<\cdots<b_{2 n}<b_{2 n+1}=b
\end{gathered}
$$

for some positive integers $m$ and $n$, such that

$$
\begin{aligned}
& r=\frac{1}{p}=0 \quad \text { on }\left(a_{2 k}, a_{2 k+1}\right) \text {, } \\
& \int_{a_{2 k}}^{a_{2 k+1}} w(x) d x \neq 0, \quad \int_{a_{2 k}}^{a_{2 k+1}} w(x) x d x \neq 0, \\
& \int_{a_{2 k}}^{a_{2 k+1}} w(x) x^{2} d x \neq 0, \quad k=0,1, \ldots, m ; \\
& r=\frac{1}{p}=0 \quad \text { on }\left(b_{2 i}, b_{2 i+1}\right) \\
& \int_{b_{2 i}}^{b_{2 i+1}} w(x) d x \neq 0, \quad \int_{b_{2 i}}^{b_{2 i+1}} w(x) x d x \neq 0, \\
& \int_{b_{2 i}}^{b_{2 i+1}} w(x) x^{2} d x \neq 0, \quad i=0,1, \ldots, n ; \\
& q=w=0 \quad \text { on }\left(a_{2 k+1}, a_{2 k+2}\right), \\
& \int_{a_{2 k+1}}^{a_{2 k+2}} r(x) d x \neq 0, \quad \int_{a_{2 k+1}}^{a_{2 k+2}} r(x) x d x \neq 0, \\
& \int_{a_{2 k+1}}^{a_{2 k+2}} r(x) x^{2} d x \neq 0, \quad k=0,1, \ldots, m-1 ; \\
& q=w=0 \quad \text { on }\left(b_{2 i+1}, b_{2 i+2}\right), \\
& \int_{b_{2 i+1}}^{b_{2 i+2}} r(x) d x \neq 0, \quad \int_{b_{2 i+1}}^{b_{2 i+2}} r(x) x d x \neq 0, \\
& \int_{b_{2 i+1}}^{b_{2 i+2}} r(x) x^{2} d x \neq 0, \quad i=0,1, \ldots, n-1 .
\end{aligned}
$$

Given (18) and (19), let

$$
\begin{gathered}
r_{k}=\int_{a_{2 k+1}}^{a_{2 k+2}} r(x) d x, \quad \widehat{r}_{k}=\int_{a_{2 k+1}}^{a_{2 k+2}} r(x) x d x, \\
\check{r}_{k}=\int_{a_{2 k+1}}^{a_{2 k+2}} r(x) x^{2} d x, \quad k=0,1, \ldots, m-1 ; \\
q_{k}=\int_{a_{2 k}}^{a_{2 k+1}} q(x) d x, \quad \widehat{q}_{k}=\int_{a_{2 k}}^{a_{2 k+1}} q(x) x d x, \\
\check{q}_{k}=\int_{a_{2 k}}^{a_{2 k+1}} q(x) x^{2} d x, \quad k=0,1, \ldots, m ;
\end{gathered}
$$




$$
\begin{array}{r}
w_{k}=\int_{a_{2 k}}^{a_{2 k+1}} w(x) d x, \quad \widehat{w}_{k}=\int_{a_{2 k}}^{a_{2 k+1}} w(x) x d x, \\
\check{w}_{k}=\int_{a_{2 k}}^{a_{2 k+1}} w(x) x^{2} d x, \quad k=0,1, \ldots, m ; \\
r_{i}^{\prime}=\int_{b_{2 i+1}}^{b_{2 i+2}} r(x) d x, \quad \widehat{r}_{i}^{\prime}=\int_{b_{2 i+1}}^{b_{2 i+2}} r(x) x d x, \\
\check{r}_{i}^{\prime}=\int_{b_{2 i+1}}^{b_{2 i+2}} r(x) x^{2} d x, \quad i=0,1, \ldots, n-1 ; \\
q_{i}^{\prime}=\int_{b_{2 i}}^{b_{2 i+1}} q(x) d x, \quad \hat{q}_{i}^{\prime}=\int_{b_{2 i}}^{b_{2 i+1}} q(x) x d x, \\
\check{q}_{i}^{\prime}=\int_{b_{2 i}}^{b_{2 i+1}} q(x) x^{2} d x, \quad i=0,1, \ldots, n ;
\end{array}
$$

$$
\begin{array}{r}
w_{i}^{\prime}=\int_{b_{2 i}}^{b_{2 i+1}} w(x) d x, \quad \widehat{w}_{i}^{\prime}=\int_{b_{2 i}}^{b_{2 i+1}} w(x) x d x, \\
\check{w}_{i}^{\prime}=\int_{b_{2 i}}^{b_{2 i+1}} w(x) x^{2} d x, \quad i=0,1, \ldots, n .
\end{array}
$$

The above mentioned notations are needed later.

Following [2] we determine the structure of the principal fundamental matrix of system (5) on which our results are based.

Lemma 7. Let (4), (18), and (19) hold. Let $\Theta(x, \lambda)=$ $\left[\theta_{i j}(x, \lambda)\right]$ be the fundamental matrix solution of the system (5) determined by the initial condition $\Theta(a, \lambda)=I$ for each $\lambda \in \mathbb{C}$ in the interval $(a, c)$. Let

$$
\begin{aligned}
& F_{k}\left(x, \lambda, a_{k}\right)=\left(\begin{array}{cccc}
1 & x-a_{k} & 0 & 0 \\
0 & 1 & 0 & 0 \\
\int_{a_{k}}^{x}(\lambda w-q)(x-t) d t & \int_{a_{k}}^{x}(\lambda w-q)(x-t)\left(t-a_{k}\right) d t & 1 & x-a_{k} \\
\int_{a_{k}}^{x}(\lambda w-q) d t & \int_{a_{k}}^{x}(\lambda w-q)\left(t-a_{k}\right) d t & 0 & 1
\end{array}\right), \\
& k=0,2, \ldots, 2 m, \\
& F_{k}\left(x, \lambda, a_{k}\right)=\left(\begin{array}{cccc}
1 & x-a_{k} & \int_{a_{k}}^{x} r(x-t) d t & \int_{a_{k}}^{x} r(x-t)\left(t-a_{k}\right) d t \\
0 & 1 & \int_{a_{k}}^{x} r d t & \int_{a_{k}}^{x} r\left(t-a_{k}\right) d t \\
0 & 0 & 1 & x-a_{k} \\
0 & 0 & 0 & 1
\end{array}\right) \\
& k=1,3, \ldots, 2 m-1 \text {. }
\end{aligned}
$$

Then for $1 \leq k \leq 2 m+1$ we have

$$
\Theta\left(a_{k}, \lambda\right)=F_{k-1}\left(a_{k}, \lambda, a_{k-1}\right) \Theta\left(a_{k-1}, \lambda\right) .
$$

And more simpler, if we let

$$
\begin{gathered}
T_{0}=F_{0}\left(a_{1}, \lambda, a_{0}\right), \\
T_{k}=F_{2 k}\left(a_{2 k+1}, \lambda, a_{2 k}\right) F_{2 k-1}\left(a_{2 k}, \lambda, a_{2 k-1}\right), \\
k=1,2, \ldots, m,
\end{gathered}
$$

then

$$
\begin{array}{r}
\Theta\left(a_{1}, \lambda\right)=F_{0}\left(a_{1}, \lambda, a_{0}\right)=T_{0}, \\
\Theta\left(a_{2 k+1}, \lambda\right)=T_{k} \Theta\left(a_{2 k-1}, \lambda\right), \\
k=1,2, \ldots, m .
\end{array}
$$

Hence we have the following formula:

$$
\Theta\left(a_{2 k+1}, \lambda\right)=T_{k} T_{k-1} \cdots T_{0}, \quad k=0,1, \ldots, m .
$$

Proof. Observe from (5) that $u_{2}$ is constant on each subinter$\operatorname{val}\left[a_{2 k}, a_{2 k+1}\right), k=0,1, \ldots, m$, where $r$ is identically zero, and thus on each of these subintervals we have that

$$
\begin{aligned}
u_{2}(x)= & u_{2}\left(a_{2 k}\right) \\
u_{1}(x)= & u_{1}\left(a_{2 k}\right)+u_{2}\left(a_{2 k}\right)\left(x-a_{2 k}\right) \\
u_{4}(x)= & u_{4}\left(a_{2 k}\right)+u_{1}\left(a_{2 k}\right) \int_{a_{2 k}}^{x}(\lambda w-q) d t \\
& +u_{2}\left(a_{2 k}\right) \int_{a_{2 k}}^{x}(\lambda w-q)\left(t-a_{2 k}\right) d t \\
u_{3}(x)= & u_{3}\left(a_{2 k}\right)+u_{4}\left(a_{2 k}\right)\left(x-a_{2 k}\right) \\
& +u_{1}\left(a_{2 k}\right) \int_{a_{2 k}}^{x}(\lambda w-q)(x-t) d t \\
& +u_{2}\left(a_{2 k}\right) \int_{a_{2 k}}^{x}(\lambda w-q)(x-t)\left(t-a_{2 k}\right) d t \\
& +u_{2}\left(a_{2 k}\right) \int_{a_{2 k}}^{x} s d t .
\end{aligned}
$$


Similarly, because $q$ and $w$ are identically zero, $u_{4}$ is constant on each subinterval $\left[a_{2 k-1}, a_{2 k}\right), k=1,2, \ldots, m-1$, so we have

$$
\begin{aligned}
u_{4}(x)= & u_{4}\left(a_{2 k-1}\right), \\
u_{3}(x)= & u_{3}\left(a_{2 k-1}\right)+u_{4}\left(a_{2 k-1}\right)\left(x-a_{2 k-1}\right), \\
u_{2}(x)= & u_{2}\left(a_{2 k-1}\right)+u_{3}\left(a_{2 k-1}\right) \int_{a_{2 k-1}}^{x} r d t \\
& +u_{4}\left(a_{2 k-1}\right) \int_{a_{2 k-1}}^{x} r\left(t-a_{2 k-1}\right) d t,
\end{aligned}
$$

$$
\begin{aligned}
u_{1}(x)= & u_{1}\left(a_{2 k-1}\right)+u_{2}\left(a_{2 k-1}\right)\left(x-a_{2 k-1}\right) \\
& +u_{3}\left(a_{2 k-1}\right) \int_{a_{2 k-1}}^{x} r(x-t) d t \\
& +u_{4}\left(a_{2 k-1}\right) \int_{a_{2 k-1}}^{x} r(x-t)\left(t-a_{2 k-1}\right) d t .
\end{aligned}
$$

We see that $u_{i}(x), i=1,2,3,4$ are piecewise continuous functions on $[a, c)$. Let $U(x)=\left[u_{1}(x), u_{2}(x), u_{3}(x), u_{4}(x)\right]^{T}$ on $[a, c)$ and set $U^{(j)}(x, \lambda)=U\left(x, \lambda ; e_{j}\right)$, where $e_{j}, j=$ $1,2,3,4$ are the standard unit vectors; then it is easy to see that $\Theta(x, \lambda)=\left[U^{(1)} U^{(2)} U^{(3)} U^{(4)}\right]$. This establishes (22).

Lemma 8. Let (4), (18), and (19) hold. Let $\Psi(x, \lambda)=$ $\left[\psi_{i j}(x, \lambda)\right]$ be the fundamental matrix solution of the system (5) determined by the initial condition $\Psi(c, \lambda)=I$ for each $\lambda \in \mathbb{C}$ in the interval $(c, b)$. Let

$$
\begin{aligned}
& \widetilde{F}_{k}\left(x, \lambda, b_{k}\right)=\left(\begin{array}{cccc}
1 & x-b_{k} & 0 & 0 \\
0 & 1 & 0 & 0 \\
\int_{b_{k}}^{x}(\lambda w-q)(x-t) d t & \int_{b_{k}}^{x}(\lambda w-q)(x-t)\left(t-b_{k}\right) d t & 1 & x-b_{k} \\
\int_{b_{k}}^{x}(\lambda w-q) d t & \int_{b_{k}}^{x}(\lambda w-q)\left(t-b_{k}\right) d t & 0 & 1
\end{array}\right), \\
& k=0,2, \ldots, 2 n \text {; } \\
& \widetilde{F}_{k}\left(x, \lambda, b_{k}\right)=\left(\begin{array}{cccc}
1 & x-b_{k} & \int_{b_{k}}^{x} r(x-t) d t & \int_{b_{k}}^{x} r(x-t)\left(t-b_{k}\right) d t \\
0 & 1 & \int_{b_{k}}^{x} r d t & \int_{b_{k}}^{x} r\left(t-b_{k}\right) d t \\
0 & 0 & 1 & x-b_{k} \\
0 & 0 & 0 & 1
\end{array}\right) \text {, } \\
& k=1,3, \ldots, 2 n-1 \text {. }
\end{aligned}
$$

Then for $1 \leq k \leq 2 n+1$ we have

$$
\Psi\left(b_{k}, \lambda\right)=\widetilde{F}_{k-1}\left(b_{k}, \lambda, b_{k-1}\right) \Psi\left(b_{k-1}, \lambda\right) .
$$

Similarly, if we let

$$
\begin{gathered}
\widetilde{T}_{0}=\widetilde{F}_{0}\left(b_{1}, \lambda, b_{0}\right), \\
\widetilde{T}_{k}=\widetilde{F}_{2 k}\left(b_{2 k+1}, \lambda, b_{2 k}\right) \widetilde{F}_{2 k-1}\left(b_{2 k}, \lambda, b_{2 k-1}\right), \\
k=1,2, \ldots, n,
\end{gathered}
$$

then

$$
\begin{gathered}
\Psi\left(b_{1}, \lambda\right)=\widetilde{F}_{0}\left(b_{1}, \lambda, b_{0}\right)=\widetilde{T}_{0}, \\
\Psi\left(b_{2 k+1}, \lambda\right)=\widetilde{T}_{k} \Psi\left(b_{2 k-1}, \lambda\right), \quad k=1,2, \ldots, n .
\end{gathered}
$$

Hence we have the following formula:

$$
\Psi\left(b_{2 k+1}, \lambda\right)=\widetilde{T}_{k} \widetilde{T}_{k-1} \cdots \widetilde{T}_{0}, \quad k=0,1, \ldots, n .
$$

Lemma 9. Let (4), (18), and (19) hold. Let $\Phi(x, \lambda)=$ $\left[\phi_{i j}(x, \lambda)\right]$ be the fundamental matrix solution of the system (5) determined by the initial condition $\Phi(a, \lambda)=I$ for each $\lambda \in \mathbb{C}$ in the whole interval $J=(a, c) \cup(c, b)$, and let $\Theta(x, \lambda)=$ $\left[\theta_{i j}(x, \lambda)\right], \Psi(x, \lambda)=\left[\psi_{i j}(x, \lambda)\right]$ be given as in Lemmas 7 and 8. Then we have that

$$
\Phi(b, \lambda)=\Psi(b, \lambda) G \Theta(c, \lambda)
$$

where $G=\left(g_{i j}\right)_{4 \times 4}=-D^{-1} C$ and $\Theta(c, \lambda)=\Theta(c-, \lambda)$ denotes the left limit at point $c$.

Proof. From the transmission conditions (3) we know that

$$
C \Phi(c-, \lambda)+D \Phi(c+, \lambda)=0
$$

and thus

$$
\Phi(c+, \lambda)=-D^{-1} C \Phi(c-, \lambda) .
$$


Let $G=-D^{-1} C$, and note that $\Theta(c, \lambda)=\Theta(c-, \lambda)=$ $\Theta\left(a_{2 m+1}, \lambda\right)=\Phi(c-, \lambda), \Psi(b, \lambda)=\Psi\left(b_{2 n+1}, \lambda\right)$ and $\Psi(c, \lambda)=I$, so we can conclude from Lemmas 7 and 8 that

$$
\Phi(b, \lambda)=\Psi(b, \lambda) G \Theta(c, \lambda)
$$

where $\Phi(b, \lambda)=\Phi\left(b_{2 n+1}, \lambda\right)$.

Remark 10. This means that the fundamental matrix solution of the system (5) with interior discontinuous transmission point determined by the initial condition $\Phi(a, \lambda)=I$ can be written as

$$
\Phi(x, \lambda)= \begin{cases}\Theta(x, \lambda), & x \in[a, c) \\ \Psi(x, \lambda) G \Theta(c-, \lambda), & x \in(c, b]\end{cases}
$$

from Lemmas 7, 8, and 9.

Note that $c=a_{2 m+1}$. The structure of $\Theta$ given in Lemma 7 yields the following.

Corollary 11. For the fundamental matrix $\Theta$ we have that

$$
\begin{aligned}
& \theta_{i j}(c, \lambda)=R_{i j} \lambda^{m}+\widetilde{\theta}_{i j}(\lambda), \quad i, j=1,2, \text { or } i, j=3,4, \\
& \theta_{i j}(c, \lambda)=R_{i j} \lambda^{m+1}+\widetilde{\theta}_{i j}(\lambda), \quad i=3,4, j=1,2, \\
& \theta_{i j}(c, \lambda)=R_{i j} \lambda^{m-1}+\widetilde{\theta}_{i j}(\lambda), \quad i=1,2, j=3,4
\end{aligned}
$$

where $R_{i j}$ are constants related to $r_{k}, \widehat{r}_{k}, \check{r}_{k}, k=0,1, \ldots, m-$ $1, w_{k}, \widehat{w}_{k}, \breve{w}_{k}, k=0,1, \ldots, m$ and the end points $a$ and $c, \widetilde{\theta}_{i j}(\lambda)$ are functions of $\lambda$, in which the degrees of $\lambda$ are smaller than $m, m+1$, or $m-1$, respectively. For example, $\theta_{11}(c, \lambda)=R_{11} \lambda^{m}+$ $\widetilde{\theta}_{11}(\lambda)$, so the degree of $\lambda$ in $\widetilde{\theta}_{11}(\lambda)$ is smaller than $m$.

Note that $b=b_{2 n+1}$. The structure of $\Psi$ given in Lemma 8 yields the following.

Corollary 12. For the fundamental matrix $\Psi$ we have that

$$
\begin{aligned}
& \psi_{i j}(b, \lambda)=R_{i j}^{\prime} \lambda^{n}+\widetilde{\psi}_{i j}(\lambda), \quad i, j=1,2, \text { or } i, j=3,4, \\
& \psi_{i j}(b, \lambda)=R_{i j}^{\prime} \lambda^{n+1}+\widetilde{\psi}_{i j}(\lambda), \quad i=3,4, j=1,2, \\
& \psi_{i j}(b, \lambda)=R_{i j}^{\prime} \lambda^{n-1}+\widetilde{\psi}_{i j}(\lambda), \quad i=1,2, j=3,4,
\end{aligned}
$$

where $R_{i j}^{\prime}$ are constants related to $r_{k}^{\prime}, \widehat{r}_{k}^{\prime}, \check{r}_{k}^{\prime}, k=0,1, \ldots, m-$ $1, w_{k}^{\prime}, \widehat{w}_{k}^{\prime}, \breve{w}_{k}^{\prime}, k=0,1, \ldots, m$ and the end points $c$ and $b, \widetilde{\psi}_{i j}(\lambda)$ are functions of $\lambda$, in which the degrees of $\lambda$ are smaller than $n, n+1$, or $n-1$, respectively. For example, $\psi_{11}(b, \lambda)=R_{11}^{\prime} \lambda^{n}+$ $\widetilde{\psi}_{11}(\lambda)$, so the degree of $\lambda$ in $\widetilde{\psi}_{11}(\lambda)$ is smaller than $n$.

From Lemmas 7, 8, 9, and Corollaries 11 and 12 we have the following.
Corollary 13. For the fundamental matrix $\Phi$ we have that

$$
\begin{gathered}
\phi_{i j}(b, \lambda)=\widetilde{R}_{i j} \lambda^{m+n+1}+\widetilde{\phi}_{i j}(\lambda), \\
i, j=1,2, \text { or } i, j=3,4, \\
\phi_{i j}(b, \lambda)=\widetilde{R}_{i j} \lambda^{m+n+2}+\widetilde{\phi}_{i j}(\lambda), \quad i=3,4, j=1,2, \\
\phi_{i j}(b, \lambda)=\widetilde{R}_{i j} \lambda^{m+n}+\widetilde{\phi}_{i j}(\lambda), \quad i=1,2, j=3,4,
\end{gathered}
$$

where $\widetilde{R}_{i j}$ are constants related to $r_{k}, \widehat{r}_{k}, \check{r}_{k}, k=0,1, \ldots, m-1$, $w_{k}, \widehat{w}_{k}, \breve{w}_{k}, k=0,1, \ldots, m, r_{k}^{\prime}, \widehat{r}_{k}^{\prime}, \check{r}_{k}^{\prime}, k=0,1, \ldots, n-1$, $w_{k}^{\prime}, \widehat{w}_{k}^{\prime}, \breve{w}_{k}^{\prime}, k=0,1, \ldots, n$, the transmission matrix $G$, and the end points $a$ and $b . \widetilde{\phi}_{i j}(\lambda)$ are functions of $\lambda$, in which the degrees of $\lambda$ are smaller than $m+n+1, m+n+2$, or $m+n$, respectively. For example, $\phi_{11}(b, \lambda)=\widetilde{R}_{11} \lambda^{m+n+1}+\widetilde{\phi}_{11}(\lambda)$, so the degree of $\lambda$ in $\widetilde{\phi}_{11}(\lambda)$ is smaller than $m+n+1$.

Now we construct regular fourth-order problems with transmission conditions which have at most $3(m+n+1)+1$ eigenvalues for positive $m, n \in \mathbb{N}$.

Theorem 14. Let $m, n \in \mathbb{N}$, and let (4), (18), and (19) hold. Then the fourth-order problem (1)-(3) has at most $3(m+n+$ 1) +1 eigenvalues.

Proof. Note that $\Delta(\lambda)=\operatorname{det}[A+B \Phi(b, \lambda)]$, where $\Phi(b, \lambda)=$ $\left[\phi_{i j}(b, \lambda)\right]$. From Lemma 5 and Corollary 13 we know that the characteristic function $\Delta(\lambda)$ is a polynomial of $\lambda$ and has the form of (13). We denote the maximum of degree of $\lambda$ in $\phi_{i j}(b, \lambda)$ by $d_{i j}, 1 \leq i, j \leq 4$, and by Corollary 13 the maximum of degree of $\lambda$ in the matrix $\Phi(b, \lambda)$ can be written as the following matrix:

$$
\left(d_{i j}\right)=\left(\begin{array}{cccc}
m+n+1 & m+n+1 & m+n & m+n \\
m+n+1 & m+n+1 & m+n & m+n \\
m+n+2 & m+n+2 & m+n+1 & m+n+1 \\
m+n+2 & m+n+2 & m+n+1 & m+n+1
\end{array}\right)
$$

In terms of (13) and (41), we conclude that the maximum of the degree of $\lambda$ in $\Delta(\lambda)$ is $3(m+n+1)+1$. Thus from the fundamental theorem of algebra, $\Delta(\lambda)$ has at most $3(m+n+$ $1)+1$ roots. This implies that the fourth-order problem (1)-(3) has at most $3(m+n+1)+1$ eigenvalues.

If the BCs are separated self-adjoint boundary conditions given as (9), we can get the following corollary.

Corollary 15. Let $m, n \in \mathbb{N}$, and let (2), (18), and (19) hold. Then the fourth-order problem (1)-(3) with separated selfadjoint boundary conditions (9) has at most $2(m+n+2)$ eigenvalues.

Proof. From Corollaries 6, 13, and (41) we know that the maximum of degree of $\lambda$ in characteristic function $\Delta(\lambda)$ is $2(m+n+2)$; hence, from the fundamental theorem of algebra, $\Delta(\lambda)$ has at most $2(m+n+2)$ roots and the result follows. 
Remark 16. In the end we remark on how the number of eigenvalues depend on the order of the equation, partition of the domain interval, and the boundary conditions (including the transmission conditions). In fact, the characteristic function $\Delta(\lambda)$ can be written as $\Delta(a, b, A, B, C, D, 1 / p, q, w, \lambda)$ to highlight its dependence on these quantities [15]. Here for fixed $a, b, A, B, C, D, 1 / p, q, w, \Delta(\lambda)$ is a polynomial function of $\lambda$, hence, any quantity does have influence on the order of the characteristic polynomial $\Delta(\lambda)$. As the number of eigenvalus $3(m+n+1)+1$ for example, it can be written as $(l-1)(m+n+1)+1$, where $l$ denotes the order of the equation, $m$ and $n$ are related with the partition of $(a, c)$ and $(c, b)$, respectively, and the influences from $\mathrm{BCs}$ or transmission conditions can be seen from Theorem 14 and its corollary or Lemma 9.

\section{Conflict of Interests}

The authors declare that there is no conflict of interests regarding the publication of this paper.

\section{Acknowledgments}

This work was supported by the National Natural Science Foundation of China (Grant no. 11301259 and Grant no. 11161030), Natural Science Foundation of Inner Mongolia (Grant no. 2013MS0105), and a Grant-in-Aid for Scientific Research from Inner Mongolia University of Technology (Grant no. ZD201310).

\section{References}

[1] F. V. Atkinson, Discrete and Continuous Boundary Problems, Academic Press, New York, NY, USA, 1964.

[2] Q. Kong, H. Wu, and A. Zettl, "Sturm-Liouville problems with finite spectrum," Journal of Mathematical Analysis and Applications, vol. 263, no. 2, pp. 748-762, 2001.

[3] Q. Kong, H. Volkmer, and A. Zettl, "Matrix representations of Sturm-Liouville problems with finite spectrum," Results in Mathematics, vol. 54, no. 1-2, pp. 103-116, 2009.

[4] J.-J. Ao, J. Sun, and M.-Z. Zhang, "The finite spectrum of SturmLiouville problems with transmission conditions," Applied Mathematics and Computation, vol. 218, no. 4, pp. 1166-1173, 2011.

[5] J.-J. Ao, J. Sun, and M.-Z. Zhang, "The finite spectrum of Sturm-Liouville problems with transmission conditions and eigenparameter-dependent boundary conditions," Results in Mathematics, vol. 63, no. 3-4, pp. 1057-1070, 2013.

[6] J.-J. Ao, J. Sun, and M.-Z. Zhang, "Matrix representations of Sturm-Liouville problems with transmission conditions," Computers \& Mathematics with Applications, vol. 63, no. 8, pp. 1335-1348, 2012.

[7] J.-J. Ao and J. Sun, "Matrix representations of Sturm-Liouville problems with eigenparameter-dependent boundary conditions," Linear Algebra and Its Applications, vol. 438, no. 5, pp. 2359-2365, 2013.

[8] J.-J. Ao, J. Sun, and A. Zettl, "Matrix representations of fourth order boundary value problems with finite spectrum," Linear Algebra and Its Applications, vol. 436, no. 7, pp. 2359-2365, 2012.
[9] J.-J. Ao, J. Sun, and A. Zettl, "Equivalence of fourth order boundary value problems and matrix eigenvalue problems," Results in Mathematics, vol. 63, no. 1-2, pp. 581-595, 2013.

[10] J.-J. Ao, F.-Z. Bo, and J. Sun, "Fourth order boundary value problems with finite spectrum," submitted to Applied Mathematics and Computation.

[11] P. A. Binding, P. J. Browne, and B. A. Watson, "Inverse spectral problems for Sturm-Liouville equations with eigenparameter dependent boundary conditions," Journal of the London Mathematical Society. Second Series, vol. 62, no. 1, pp. 161-182, 2000.

[12] Z. Akdoğan, M. Demirci, and O. Sh. Mukhtarov, "Green function of discontinuous boundary-value problem with transmission conditions," Mathematical Methods in the Applied Sciences, vol. 30, no. 14, pp. 1719-1738, 2007.

[13] Q. X. Yang and W. Y. Wang, "A class of fourth order differential operators with transmission conditions," Iranian Journal of Science and Technology A, vol. 35, no. 4, pp. 323-332, 2011.

[14] X.-Y. Zhang and J. Sun, "The determinants of fourth order dissipative operators with transmission conditions," Journal of Mathematical Analysis and Applications, vol. 410, no. 1, pp. 5569, 2014.

[15] A. Zettl, Sturm-Liouville Theory, vol. 121 of Mathematical Surveys and Monographs, American Mathematical Society, 2005.

[16] W. N. Everitt and D. Race, "On necessary and sufficient conditions for the existence of Carathéodory solutions of ordinary differential equations," Quaestiones Mathematicae, vol. 2, no. 4, pp. 507-512, 1976.

[17] B. Chanane, "Accurate solutions of fourth order Sturm-Liouville problems," Journal of Computational and Applied Mathematics, vol. 234, no. 10, pp. 3064-3071, 2010.

[18] L. Greenberg and M. Marletta, "Numerical methods for higher order Sturm-Liouville problems," Journal of Computational and Applied Mathematics, vol. 125, no. 1-2, pp. 367-383, 2000.

[19] A. Wang, J. Sun, and A. Zettl, "Characterization of domains of self-adjoint ordinary differential operators," Journal of Differential Equations, vol. 246, no. 4, pp. 1600-1622, 2009.

[20] X. Hao, J. Sun, A. Wang, and A. Zettl, "Characterization of domains of self-adjoint ordinary differential operators II," Results in Mathematics, vol. 61, no. 3-4, pp. 255-281, 2012. 


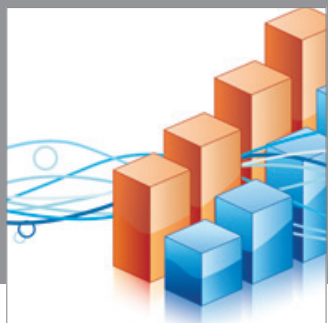

Advances in

Operations Research

mansans

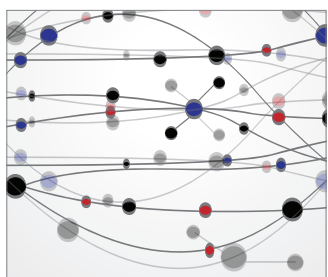

The Scientific World Journal
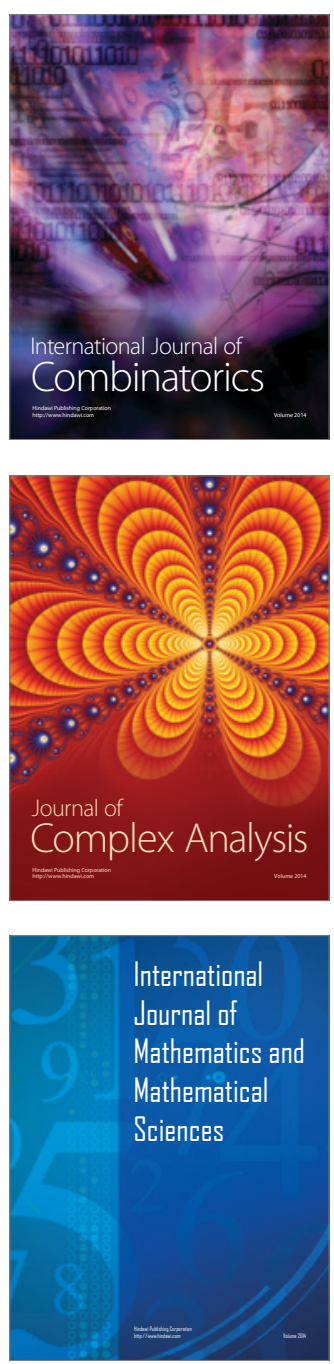
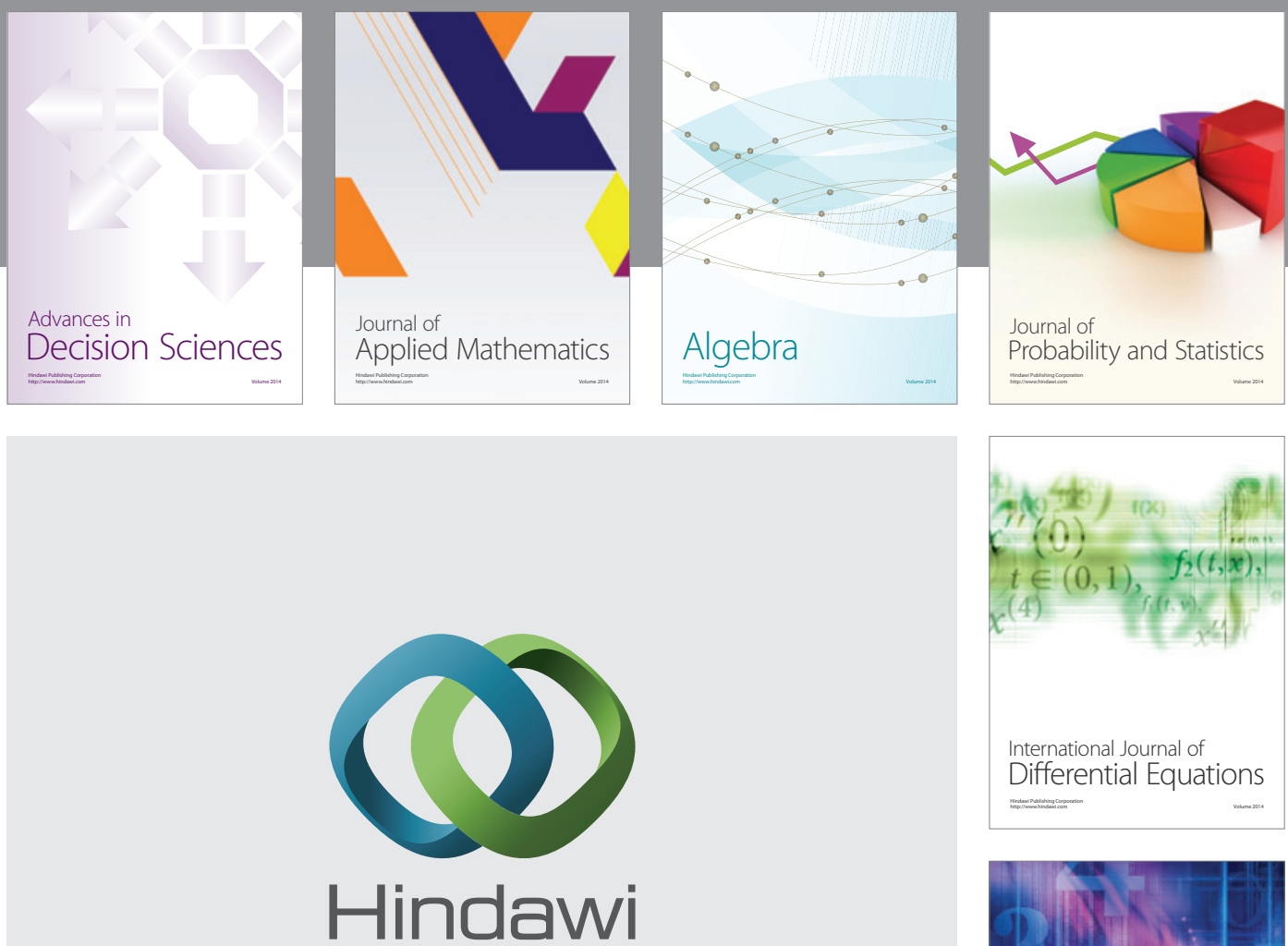

Submit your manuscripts at http://www.hindawi.com
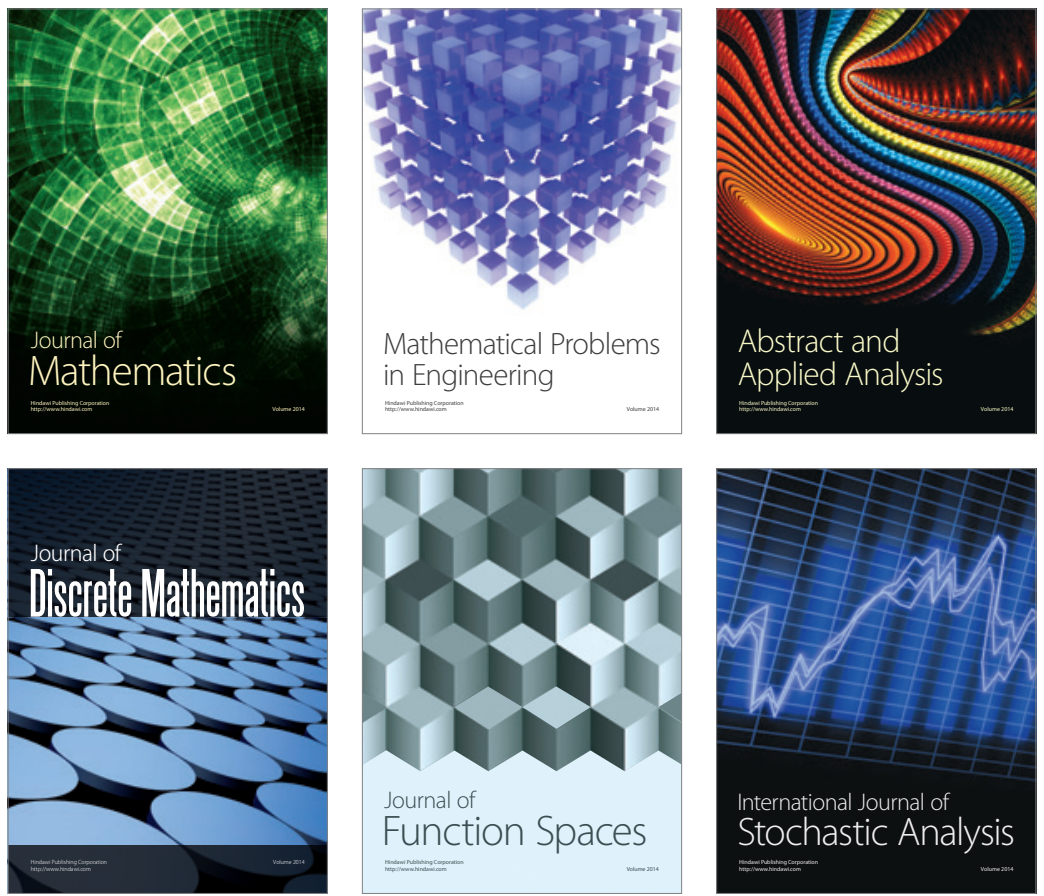

Journal of

Function Spaces

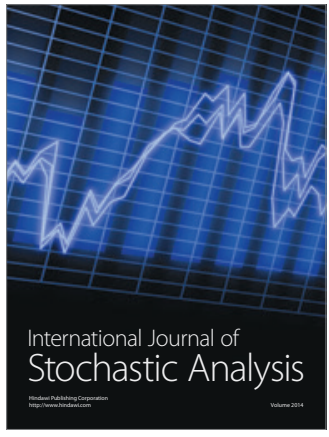

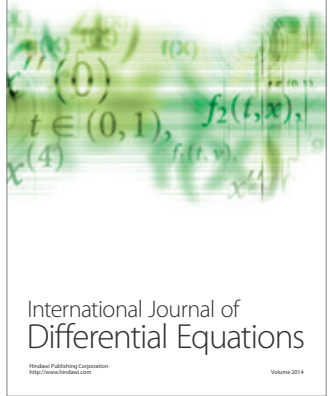
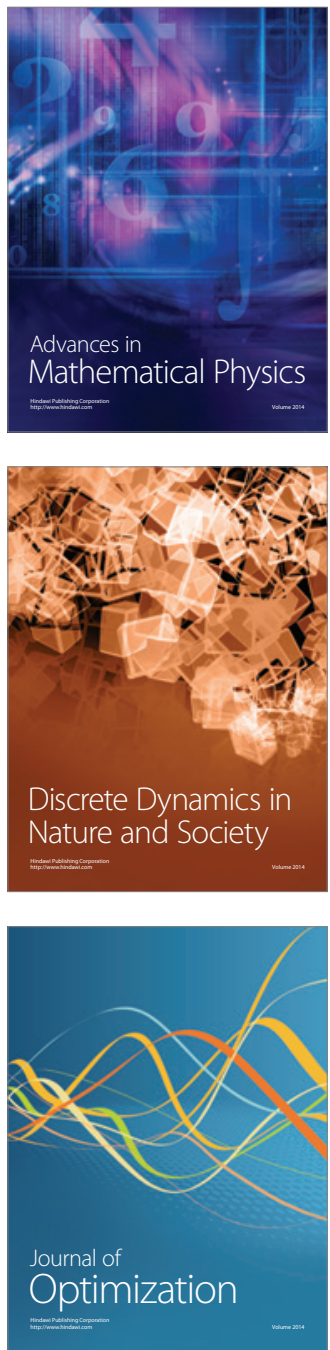